de la dissolution dans l'eau de matières albuminoïdes, qui se combinent à l'alcali, une partie d'ailleurs précipite lors de la neutralisation. Mais en remplaçant l'eau par l'alcool, peut-être y aurait-il une différence due à la grosseur des grains comme nous le faisions remarquer plus haut.

On ne dose pas ainsi l'acidité due aux acides gras de la matière grasse que retient toujours un peu la easéine industrielle. Cette acidité est faible. Elle se trouve comptée dans l'acidité totale.

Pour le dosage de cette acidité totale, nous préférons dissoudre la caséine, à froid, dans un excès connu de liqueur basique titrée et neutraliser en retour l'excès par une liqueur acide titrée. C'est ce qui a été préconisé par ULEx et par BRoWNE (The proximate analysis of commercial caseins. J. Ind. Eng. Chem. 11 (1919) (1019) on évite ainsi le chauffage done une plus grande chance d'hydrolise et l'addition successive d'alcali.

Par contre le virage du rose à l'incolore est peut-être moins net. Pour ce dosage nous préférons laisser gonfler quelque temps la caséine dans l'eau avant d'ajouter l'alcali, la dissolution en est grandement facilitée, elle est plus rapide et on peut ainsi mettre un faible excès d'alcali ce qui rend minimes les craintes d'hydrolyse.

C'est en effet une eritique que l'on peut faire à cette méthode.

Quoi qu'il en soit, que cherche l'industriel dans l'analyse des caséines commerciales ?

Le fabricant veut une méthode qui lui donne des résultats comparables, lui permettant de voir les variations relatives de sa fabrication et par là de la surveiller. Cela lui importe plus que l'exactitude, en valeur absolue, des résultats.

C'est aussi ce que désire le consommateur qui doit comparer entre eux plusieurs échantillons de caséine industrielle pour choisir la meilleure.

La chimie analytique n'est pas toujours la science aussi exacte que l'on veut bien dire, il faut bien entendu choisir les méthodes pratiques qui donnent les résultats les plus justes, mais il faut surtout que les méthodes soient précisées, unifiées afin que les résultats analytiques soient toujours comparables entre eux. Nous espérons que ce sera l'œuvre du prochain Congrès de l'Association française pour l'Avancement des Sciences, à la Rochelle.

Nous serions très heureux si ces quelques réflexions pouvaient y aider.

\title{
L'ACIDITÉ DE LA CASÉINE INDUSTRIELLE
}

\author{
par A. TAPERNOUX
}

Agrégé de Chimie à l'Ecole Vétérinaire de Lyon

L'industrie prépare actuellement d'une façon courante deux variétés de caséine qui, au point de vue théorique, ne représentent pas tout à fait la même substance chimique :

$10 \mathrm{La}$ caséine lactique;

$2^{\circ}$ La caséine à la présure. 
Je laisse volontairement de côté les caséines dites aux acides qui sont précipitées par d'autres acides que l'acide lactique.

La caséine lactique est obtenue par l'acidification progressive du lait écrémé jusqu’à coagulation spontanée. La caillebotte est ensuite purifiée par lavages, et peut subir différents traitements purificateurs, puis elle est séchée.

La caséine à la présure est préparée, comme son nom l'indique, par la eoagulation du lait écrémé au moyen de la présure. Les mêmes opérations succèdent pour la purification.

Il est évident que la valeur marchande d'une caséine dépend de son état de pureté et l'acidité peut donner à cet égard des renseignements utiles.

Et d'abord, il est utile de s'entendre lorsqu'on parle de l'acidité de la caséine. Si nous faisons gonfler dans de l'eau de la caséine extrêmement pure, comme il est possible d'en obtenir au laboratoire, et si nous ajoutons au mélange quelques gouttes de phtaléine du phénol, puis de la soude titrés, nous voyons que lorsqu'un premier virage a été obtenu, une simple agitation suffirait à faire disparaître la teinte rosée. La caséine fixe la base en donnant une solution de caséinate, ce qui va de soi, la caséine ayant une acidité propre. Ces propriétés spéciales de la caséine, très intéressantes au point de vue scientifique, ne constituent pas ce que nous dénommons acidité de la caséine. Par là, nous voulons entendre seulement la mesure de l'acidité due aux impuretés de la caséine, impuretés parmi lesquelles l'acide lactique tient la première place.

C'est la mesure de cette acidité, prise au sens où nous l'entendons, qui nous renseignera sur la proportion de matières étrangères que renferme une caséine, et sur les soins plus ou moins grands apportés à la fabrication.

Dans un article intéressant, A. Chollet (1) a fixé une méthode de dosage de l'acidité des caséines industrielles. Le principe de cette méthode consiste à doser au moyen de soude Dornic, l'acidité totale à chaud de 2 gr. de caséine. L'auteur dose donc l'acidité propre de la caséine et l'acidité due aux impuretés, sans séparer ce qui revient exactement à chaque élément d'évaluation.

1 Cette méthode qui peut pratiquement rendre des services est néanmoins sujette aux critiques suivantes :

$1^{0}$ Il n'est pas démontré que les caséines-présures présentent une acídité propre de même ordre de grandeur que les caséines lactiques. Nous n'avons pas à faire dans les deux cas, comme je l'ai déjà dit, à la même substance.

$2^{\circ}$ Il est diffisile d'apprécier par cette méthode ce qui revient aux impuretés de la caséine industrielle.

(1) Choll 3t. Le Lait, 1928, p. 21. 
C'est pourquoi nous avons, de notre côté, cherché un autre procédé d'évaluation et voici la technique à laquelle nous nous sommes provisoirement arrêté :

Technique. - On pèse exactement un gramme de caséine préalablement pulvérisée au moulin à café, de façon à obtenir toujours un grain identique à lui-même. Introduire la quantité pesée dans une capsule de platine bien propre (une capsule de nickel peut suffire) et mettre macérer avec $10 \mathrm{~cm}^{3}$ d'eau distillée, en chauffant légèrement et en agitant avec un agitateur de verre. Laisser déposer la easéine et décanter le premier liquide dans un tube à essai. Ajouter $10 \mathrm{~cm}^{3}$ d'eau distillée sur la caséine et répéter la même opération en recueillant le liquide décanté dans le même tube à essai.

Renouveler une troisième fois la même opération. Ajouter au liquide trois gouttes de phtaléine du phénol et titrer avec la soude Dornic.

Expression des résultats. - Nous avons toujours obtenu le même résultat pour la même caséine. C'est la condition nécessaire et suffisante pour permettre une comparaison.

Supposons qu'on ait trouvé $12^{\circ}$ Dornic. On rapportera le résultat à 100 gr. de caséine en disant que cette caséine renferme 1 gr., 2 d'acide lactique pour cent. S'il s'agissait d'une easéine aux acides, on pourrait exprimer le résultat en acide sulfurique en multipliant $1 \mathrm{gr}, 2$ par 0,54.

Dans le cas d'acide ehlorhydrique, il suffirait de multiplier 1 gr. 2 par 0,41 .

Résultats. - En employant cette technique, les caséines ẩ la présure donnent des résultats extrêmement faibles : $1 / 10$ de centimètre cube de soude Dornic suffit à faire rougir la phénolphtaléine.

Quant aux caséines lactiques, l'acidité est essentiellement variable en moyenne de 6 à $12^{\circ}$ Dornic. Ces résultats varient évidemment avec la plus ou moins grande perfection des opérations de lavage.

\section{ANALYSE DES CASÉINES INDUSTRIELLES}

\section{par André CHOLLET,}

Ingénieur Agronome, Professeur à 1'Ecole de Laiterie de Surgères

Nous nous contenterons de rappeler les méthodes que nous employons au laboratoire de l'Ecole de Laiterie de Surgères (ces méthodes avaient été mises au point par DoRNIC et DAIRE qui les avaient publiées en 1909, dans la Revue Générale du Lait, p. 328) seule la méthode de dosage de l'acidité que nous n'employons que depuis deux ans a été exposée dans Le Lait, 1928, page 21.

Prélèvement de l'échantillon. - Il est nécessaire de prélever un échantillon moyen sur le contenu de plusieurs sacs d'une même marchandise, et en différents points d'un même sac ; les cannes-sondes employées dans le commerce des grains sont très utiles dans ce but. La caséine étant douée 